\title{
Case Report of Osteopoikilosis: Sparse Cause of Bone Pain and Mimicker of Metastasis on Radiographs
}

\author{
Sameer R. Panchal ${ }^{1}$, Sangeet Gawhale ${ }^{1}$, Nadir Z. Shah ${ }^{1}$, Tapas Mohanty ${ }^{1}$
}

\section{Learning Point of the Article:}

The purpose of this case report is to enlighten the presentation of OPK, being an extremely rare condition and always keeping this in mind as an infrequent cause of bone pain and differential for osteoblastic metastasis.

\section{Abstract}

Introduction: Osteopoikilosis (OPK) is an extremely rare benign condition with autosomal dominant inheritance characterized by sclerosing bony dysplasia with multiple benign enostoses. It is characterized by symmetrically distributed numerous, small, well-defined, homogenous circular or ovoid radiodensities clustered in epiphysis and metaphysis of long bones in periarticular region, and in some cases diffusely present throughout axial and appendicular skeleton. There is no age and sex predilection; age at the time of diagnosis ranges from 15 to 60 years. It is usually asymptomatic but rarely in $15-20 \%$ patients slight juxta-articular pain and joint effusions can be seen. These are incidental radiological findings in most of the cases, also sometimes confused as bony metastasis. There are no specific clinical features; histological features are similar to bony island and it may be associated with connective tissue disorders, synovial osteochondromatosis, and a rare bone condition melorheostosis.

Case Report: We present a case of OPK in a 32-year-old male with bilateral hip and shoulder pain, based on the available literature and focus on clinical significance, due to its mimicking capability of other more severe conditions such as bone metastases and an extremely uncommon cause ofbone pain.

Conclusion: OPK is an uncommon hereditary condition involving juxta-articular region of long bones with intricate etiopathogenesis, often discovered incidentally on radiographs. It is characterized by multiple, symmetrical ovoid radiodensities, and in most instances confused with osteoblastic metastasis. This concludes that OPK is a condition that should be kept in mind to avoid misdiagnosis, in particular osteoblastic metastasis and undue distress to both the patients and doctors.

Keywords: Osteopoikilosis, melorheostosis, bone metastases

\section{Introduction}

Osteopoikilosis (OPK) also known as osteopathia condensans disseminata or spotted bone disease is a rare hereditary autosomal dominant condition characterized by sclerosing bone dysplasia. First detailed description of this rare pathological condition was made by Albers-Schonberg in 1915 [1]. It appears with variable onset in both sexes regardless the age but preferably adult males. Incidence of this condition is not known since the disease is of little clinical importance and constitutes an incidental radiological finding. Until 1971, Szabo was able to find only 300 cases in the literature and claimed that the incidence of OPK is 1 in every 50,000 subjects [2]. There have also been reports of sporadic cases. Diagnosis is made by incidental classical radiological findings in patients who come in for treatment for other medical conditions such as fractures and arthritis. There is an abnormality in endochondral bone maturation process and collagen regulation, which leads to the formation of trabeculae along the lines of stress.

Heterozygous LEMD3 gene mutation is been shown to be the genetic cause of the disease. Furthermore, there are findings of some patients developing superficial skin lesions and/or subcutaneous foci of dermatofibrosis. Such a combination of

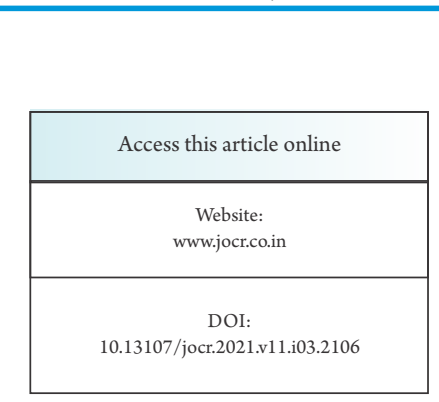

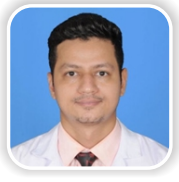

Dr. Sameer R. Panchal

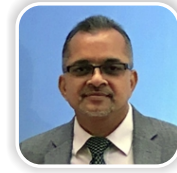

Dr. Sangeet Gawhale



Dr. Nadir Z. Shah

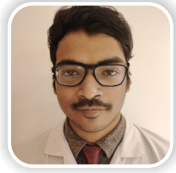

Dr. Tapas Mohanty

'Department of Orthopaedics, Grant Medical College and Sir JJ Group of Hospitals, Byculla, Mumbai, Maharashtra, India.

Address of Correspondence:

Dr. Sameer R. Panchal,

Department of Orthopaedics, Grant Government Medical College and Sir JJ Group of Hospitals, Byculla, Mumbai - 400 008, Maharashtra, India.

E-mail: panchalsameer1992@gmail.com

Journal of Orthopaedic Case Reports | pISSN 2250-0685 | eISSN 2321-3817 | Available on www.jocr.co.in | doi:10.13107/jocr.2021.v11.i03.2106 This is an Open Access article distributed under the terms of the Creative Commons Attribution Non-Commercial License (http://creativecommons.org/licenses/by-nc/3.0) which permits unrestricted non-commercial use, distribution, and reproduction in any medium, provided the original work is properly cited. 


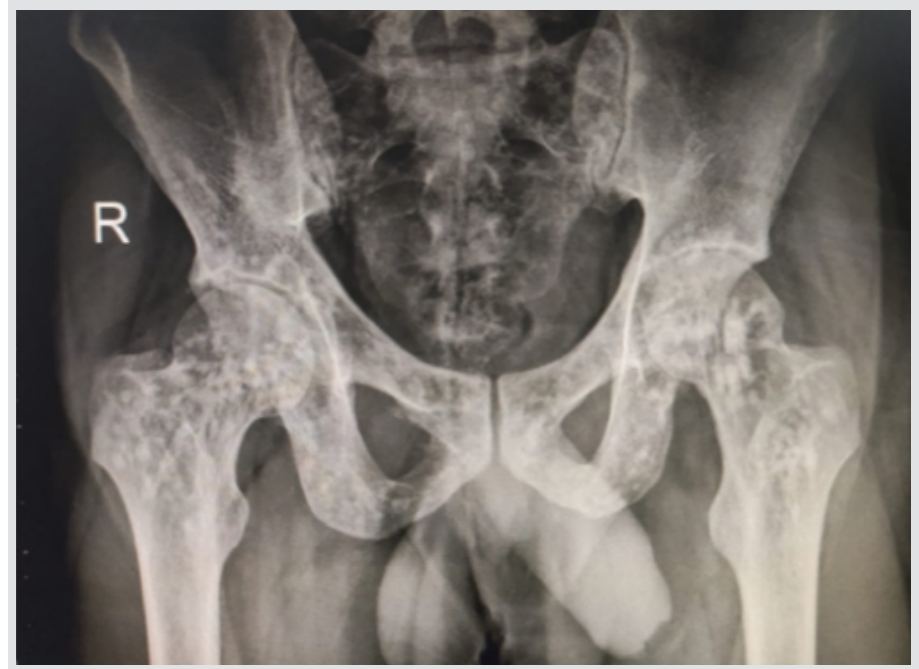

Figure 1: Anteroposterior radiograph of pelvis demonstrating dense round sclerotic lesions in both hips, pubic rami, and proximal femur.

OPK and skin lesions is known as Buschke-Ollendorff syndrome [3]. OPK is also seen with a more severe bone disease named as melorheostosis in which asymmetric depositions of dense compact bone are present on the periosteal and endosteal surface of long bones and lead to bone deformation and ossification of soft tissue in joint proximity and compression of adjacent nerves and vessels which is painful [4]. In familial or congenital OPK, there is intermittent pain in hands and feet of the patients. The onset of the symptoms ranging from 15 to 26 years of age [5]. The bony lesions are less in a younger age group and hence can be missed early in life. Histologically, the bone lesions are numerous dense bone trabeculae, which are slightly thicker than the normal within the spongiosa.

\section{Case Report}

A 32-year-old male, software engineer by occupation presented to the outpatient department with complaints of bilateral hip and shoulder pain for the past 2 months. Pain was insidious in onset, more sort of mechanical in nature which was aggravated on exertion and relieved on rest and analgesics. The patient had no history of past medical illness, significant family history and was absolutely asymptomatic before this presentation.

Physical examination revealed that range of motion of all joints was within normal limits with no neurovascular deficit. No skin

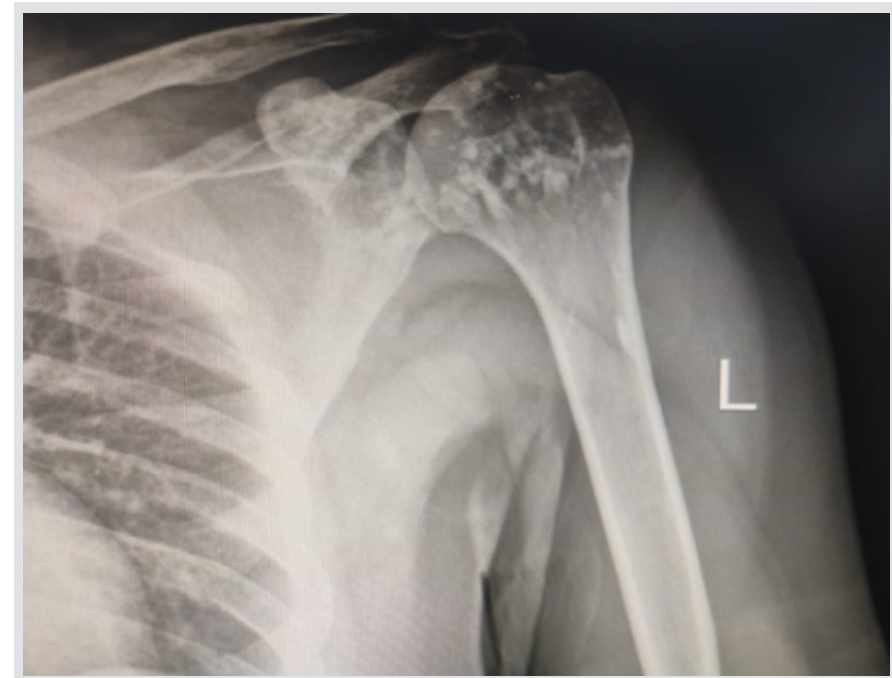

Figure 2: Anteroposterior view of the left shoulder demonstrating sclerotic lesions in proximal humerus.

lesions and palpable lymphadenopathy were detected.

Blood investigations such as complete blood count, erythrocyte sedimentation rate, C-reactive protein, kidney and liver function tests, serum calcium, phosphorus, magnesium, alkaline phosphatase, Vitamin D3, and parathyroid hormone levels were normal.

\section{Radiological examination}

Pelvis radiograph revealed numerous small foci of bony sclerosis on the femoral heads and acetabulum (Fig. 1). Bilateral shoulder anteroposterior view also revealed similar findings involving humeral heads and glenoid fossa adding to our surprise (Fig. 2). Complete skeletal survey was advised in view of such suspicious lesions which suggested of similar lesions scattered over axial and appendicular skeleton, particularly in periarticular region.

Computerized tomography (CT) examination of pelvis confirmed small subcentimeter sized round sclerotic bone islands in both iliac wings, sacrum, and around hip joints mimicking metastasis (Fig. 4, 5). OPK was suspected from these findings and magnetic resonance imaging (MRI) pelvis with both hips was advised to confirm the diagnosis.

MRI of pelvis confirmed multiple small areas of altered signal in

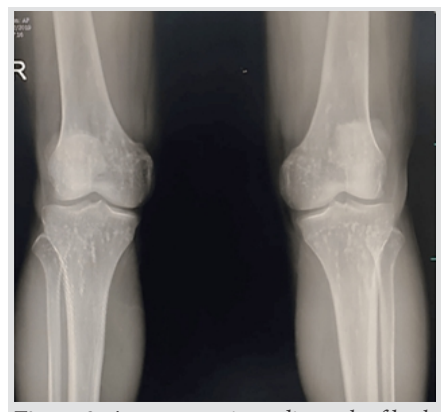

Figure 3: Anteroposterior radiograph of both knees showing osteopoikilotic lesions.
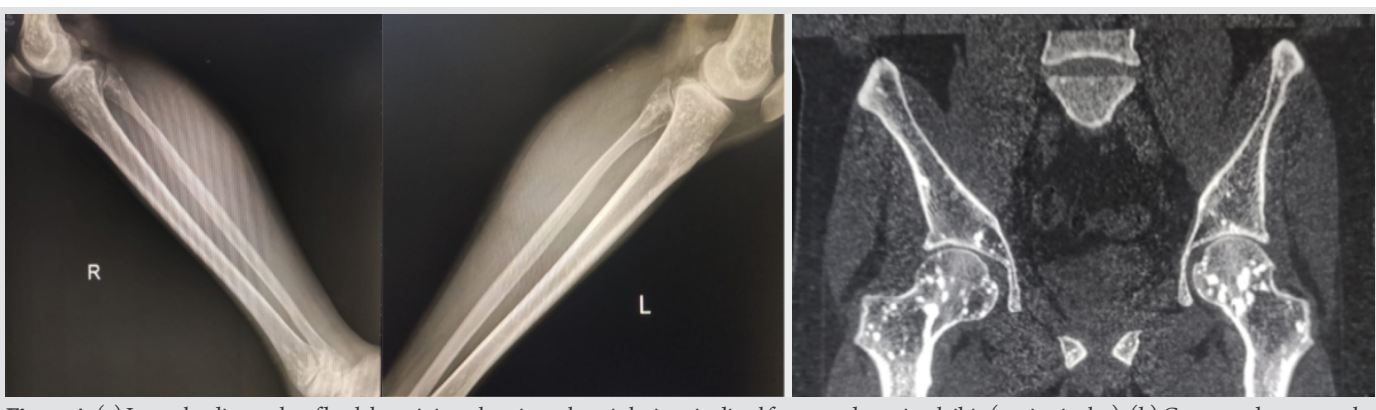

Figure 4: (a) Lateral radiographs of both knee joints showing sclerotic lesions in distal femur and proximal tibia (periarticular). (b) Computed tomography scan coronal view demonstrating sclerotic foci of variable size. 


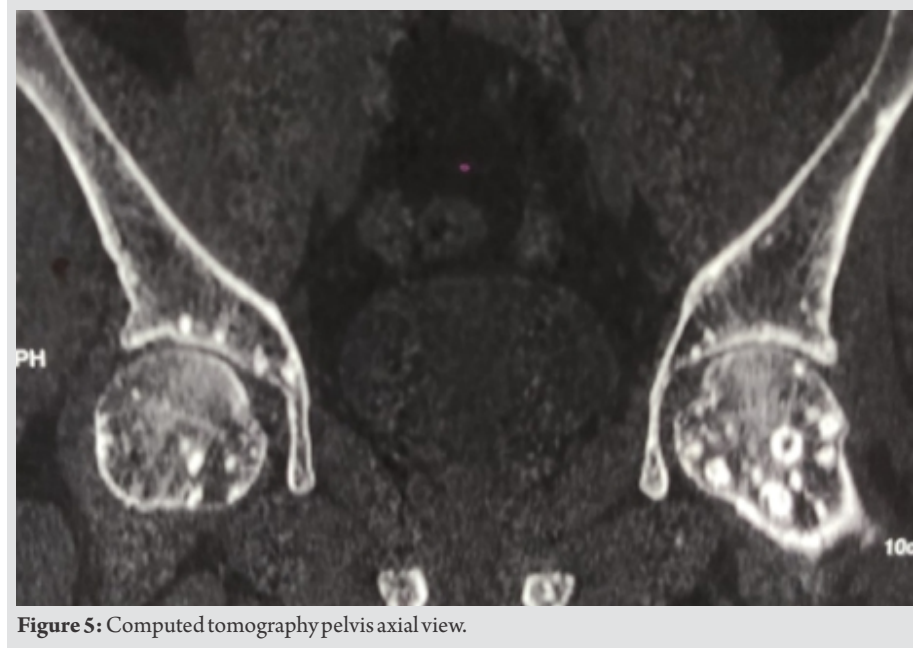

the femoral head, neck, intertrochanteric region of the femur bilaterally, as well as in the innominate bones and sacrum which appear hypointense on T1, T2 images, and STIR images (Fig. 6, 7). With these clinicoradiological findings, we concluded the diagnosis of OPK.

Conservative management with nonsteroidal antiinflammatory drugs (NSAIDs) and rest resulted in improved pain management. At present, the patient requires analgesics and rest on and off to maintain his daily activities.

\section{Discussion}

OPK is a rare lifelong heritable disease characterized by osteosclerotic dysplasia of bones with complex etiopathogenesis [1]. Overall incidence of OPK is estimated to be 1:50,000 [2]. Genetic studies suggest autosomal dominant inheritance with possible increasing penetrance [3]. Male-tofemale ratio is 3:2 $[3,6]$. Benli et al. noticed that most frequent sites for OPK appearances were the phalanges (100\%), carpal bones (97.4\%), metacarpals (92.3\%), phalanges of the foot (87.2\%), metatarsals $(84.4 \%)$, tarsal bones $(84.6 \%)$, pelvis (74.4\%), femur $(74.4 \%)$, radius and ulna $(66.7 \%)$, sacrum (58.9\%), humerus (28.2\%), tibia (20.5\%), and fibula (12.8\%) [5]. It is diagnosed by radiographic investigations characterized by numerous, symmetric, homogenous, and circular or ovoid

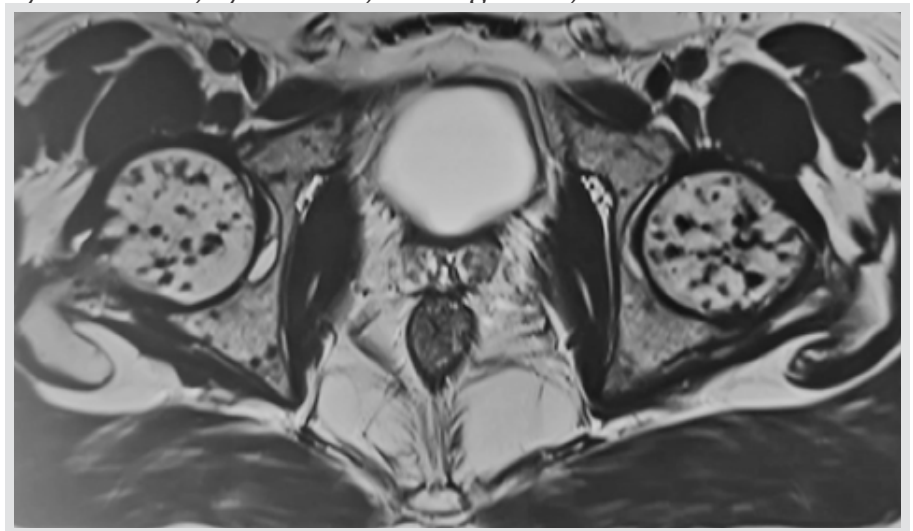

Figure 7: Magnetic resonance imaging (T2) pelvis with both hips axial view.

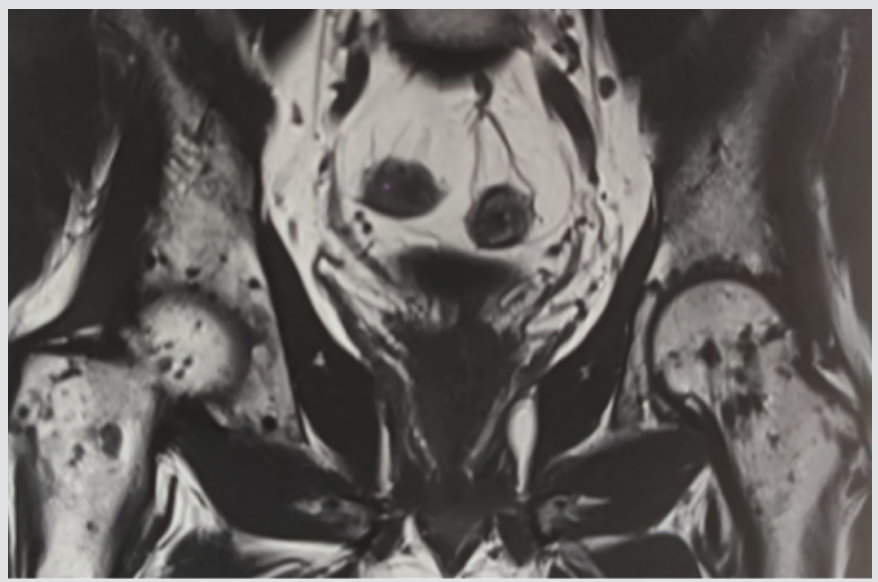

Figure 6: Magnetic resonance imaging pelvis (T2-weighted image) coronal view showing multiple hypointense signal in the femurhead, neck, and intertrochanteric region.

bony islands [7]. Patients are usually asymptomatic with laboratory tests and bone scan normal in most cases, although around $20 \%$ of patients may have mild articular pain and joint effusion. The main complaint in our case which compelled him to seek medical attention was persistent pain in affected areas. Some hypothesis had been made to explain the mechanisms of pain in OPK. Increased bone metabolism at the location of the lesion, irritation of joint capsule attachment by sclerotic areas, and increased intraosseous pressure due to venous stasis at the affected areas can produce joint pain [7]. OPK requires no treatment other than education and reassurance for patients but should be identified to prevent unnecessary invasive testing and distress. Fracture is a rare presentation of the disease.

There is no consensus on literature about the treatment. NSAIDs are often used to alleviate pain. Analgesics such as acetaminophen and opioids can also be used. Rare active lesions have been treated with bisphosphonate therapy, but the results are controversial. Our patient was managed conservatively with NSAIDs and avoiding strenuous activities.

The differential diagnosis includes osteoblastic metastasis, mastocytosis, tuberous sclerosis, and synovial chondromatosis. In most instances, it is confused with osteoblastic metastasis; uniform size, symmetrical periarticular distribution (longitudinally in the metaphysis aligning parallel to surrounding trabeculae) with normal bone scan or slightly increased activity, normal alkaline phosphatase support diagnosis of OPK in contrast asymmetrical, axial skeleton involvement, bony destruction, and positive bone scintigraphy are main diagnostic features of osteoblastic metastases [8]. Radiologic signs of OPK are similar to our finding. CT scan and MRI findings observed in our case are same as those described in the literature. Several developmental dysplasias coexisting with OPK have been reported by many authors as case reports, which suggest that OPK is a bony manifestation of a generalized fibroproliferative disease, thus in a case of OPK always consider associated fibroproliferative disorders [9]. The cooccurrence of 
OPK and melorheostosis in the same family has been reported in a few instances [4]. Melorheostosis is characterized by a "flowing" hyperostosis of the cortex of tubular bones. These lesions are usually asymmetric: They may involve only one limb or correspond to a particular sclerotome. They are often accompanied by abnormalities of adjacent soft tissues, such as joint contractures, sclerodermatous skin lesions, muscle atrophy, hemangiomas, and lymphoedema [10]. The most common overlap syndrome in which OPK combined with other osteosclerotic disorders is a combination of melorheostosis, OPK, and osteopathia striata (type 1) [11]. Complications of OPK are rare, but cases of osteosarcoma, chondrosarcoma, and giant cell tumor have been reported although no definite association has been established $[12,13]$.

Overall prognosis for the patient is good; however, asymptomatic members of their family should be screened as some of these can develop malignant transformation [13]. Knowledge of the pathognomonic radiographic features of OPK can avoid multiple investigations as well as anxiety in patient and physician alike [14].

\section{Conclusion}

$\mathrm{OPK}$ is an asymptomatic genetic condition with benign nature in most instances but in some patients with characteristic radiological findings, the patients may present with pain. Familial occurrence of OPK in relatives of the affected person should also be considered. Knowledge of typical radiological features, differential diagnosis, and associated disorders can

\section{Clinical Message}

The purpose of this case report is to enlighten the presentation of OPK, being an extremely rare condition and always keeping this in mind as an infrequent cause of bone pain and differential for osteoblastic metastasis. Most of the patients respond to conservative management, and thus, welltimed follow-up visits of the patients are recommended to exclude other conditions mimicking OPK that may require treatment.

\section{References}

1. Mohapatra MK, Patro SS, Jajodia NK, Agarwal S. Osteopoikilosis.J Assoc Physicians India 2006;54:939.

2. Szabo AD. Osteopoikilosis in a twin. Clin Orthop Relat Res 1971;79:156.

3. Hellemans J, Preobrazhenska O, Willaert A, Debeer P, Verdonk PC, Costa T, et al. Loss-of-function mutations in LEMD3 result in osteopoikilosis, Buschke-Ollendorff syndrome and melorheostosis. Nat Genet 2004;36:12138 .

4. Debeer P, Pykels E, Lammens J, Devriendt K, Fryns JP. Melorheostosis in family with autosomal dominant osteopoikilosis: Report of a third family. Am J Med Genet 2003;119A:188-93.

5. Benli IT, Akalin S, Boysan E, Mumcu EF, Kiş M, Türkoğlu D. Epidemiological, clinical and radiological aspects of osteopoikilosis. J bone Joint Surg Br 1992;74:504-6.

6. Melnick JC. Osteopathia condensans disseminate (osteopoikilosis); study of family of 4 generations. Am J Roentgenol Radium Ther Nucl Med 1959;82:229-38.

7. Mahbouba J, Mondher G, Amira M, Walid M, Naceur B. Osteopoikilosis: A rare cause of bone pain: Caspian J
Conflict of Interest: Nil Source of Support: Nil

Consent: The authors confirm that informed consent was obtained from the patient for publication of this case report

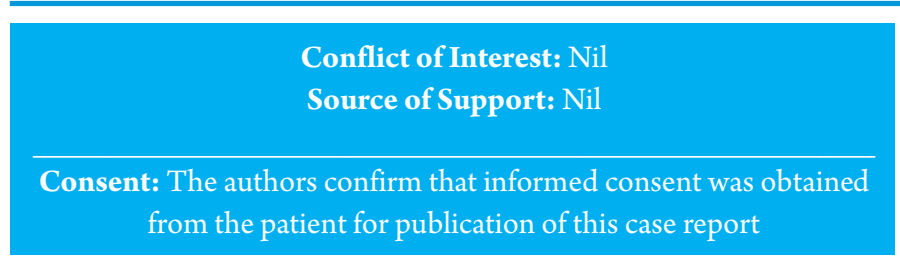

Intern Med 2015;6:177-9.

8. Krishna D, Chand S. Osteopoikilosis: A case report with review of literature. J Orthop Traumatol Rehabil 2013;6:84-6.

9. Resnick D, Niwayama G. Enostosis, hyperostosis and periostosis. In: Reznick D, Niwayama G, editors. Diagnosis of Bone and Joint Disorders. Philadelphia, PA: W.B. Saunders Company; 1988.p. 4084.

10. Rozencwaig R, Wilson MR, Mcfarland GB Jr. Melorheostosis. Am J Orthop 1997;26:83-9.

11. Alymlahi E, Chami I, Boujida NM, Bacadi D. Osteopoikilosis (OPK). Appl Radiol 2007;36:10037.

12. Hann G, Thust SC, Devereux E. Mysterious but not serious? BMJ Case Rep 2011;2011:4726.

13. Ye C, Lai Q, Zhang S, Gao T, Zeng J, Dai M. Osteopoikilosis found incidentally in a 17 years old adolescent with femoral shaft fracture-a case report. Medicine 2017;96:47.

14. Bull M, Calderbank P, Ramachandran N. A cause for concern? Osteopoikilosis found incidentally in the emergency department: A case report. Emerg Med J 2007;24:e29.

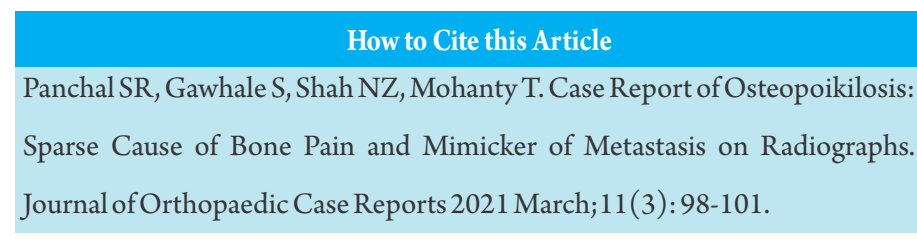

